\title{
Multiple List Learning in Adults with Autism Spectrum Disorder: Parallels with Frontal Lobe Damage or Further Evidence of Diminished Relational Processing?
}

\author{
Dermot M. Bowler · Sebastian B. Gaigg • \\ John M. Gardiner
}

Published online: 13 August 2009

(c) The Author(s) 2009. This article is published with open access at Springerlink.com

\begin{abstract}
To test the effects of providing relational cues at encoding and/or retrieval on multi-trial, multi-list free recall in adults with high-functioning autism spectrum disorder (ASD), 16 adults with ASD and 16 matched typical adults learned a first followed by a second categorised list of 24 words. Category labels were provided at encoding, retrieval, both or not at all. Both groups showed enhanced recall when labels were available during encoding or throughout the task. ASD individuals showed reduced recall of the second list and reduced clustering. Clustering and recall were correlated in both groups, which also showed similar levels of subjective organisation. The findings are discussed in relation to theories of frontal and medial temporal lobe contributions to memory in ASD.
\end{abstract}

Keywords Autism spectrum disorder - Learning · Memory $\cdot$ Free recall $\cdot$ Multi-trial $\cdot$ Clustering

\section{Introduction}

Memory in autism spectrum disorder (ASD) is characterised by a particular pattern of performance across tasks. Better performance tends to be seen on tasks such as recognition and cued recall, which provide some support at test; poorer performance is more likely on tasks, such as free recall, which do not provide such support (see Bowler and Gaigg 2008 for review). More precisely, performance on single-trial free recall tasks is diminished in individuals with some global intellectual disability (Boucher and

D. M. Bowler $(\bowtie) \cdot$ S. B. Gaigg · J. M. Gardiner

Autism Research Group, Department of Psychology, City

University, Northampton Square, London EC1V OHB, UK

e-mail: d.m.bowler@city.ac.uk
Warrington 1976), but usually intact in individuals with normal or higher levels of cognitive functioning (Bowler et al. 1997). However, both groups show diminished performance either when semantic or associative relations among the studied items can be recruited in support of recall (Hermelin and O'Connor 1967; Bowler et al. 1997, 2000; Smith et al. 2007; Tager-Flusberg 1991), or when learning and recall is tested over a sufficiently extended number of trials (Bowler et al. 2008).

The patterning of performance on supported and unsupported tasks led to the development of the Task Support Hypothesis (TSH, Bowler et al. 1997), which predicts undiminished performance by individuals with ASD on memory tasks that provide support for the retrieval of information. A similar pattern of findings has emerged in the typical aging literature where older individuals also tend to rely on greater environmental support for the retrieval of previously learned information. This is especially so when older individuals show diminished frontal lobe functioning (Craik et al. 1990). Younger individuals with frontal lobe damage also show diminished memory performance when unsupported test procedures are employed (see Baldo and Shimamura 2002 for review). This parallel between the patterning of memory findings in ASD, typically aging individuals, and frontal lobe pathology, suggests that some of the memory difficulties in ASD may, at least in part, be the result of functional abnormalities in the frontal lobes. This idea is further supported by evidence suggesting that individuals with ASD perform relatively poorly on certain executive function tasks, which are also thought to be mediated by the frontal lobes (see Hill 2004 for review).

One way of establishing the extent to which the memory profile of individuals with ASD parallels that of individuals with frontal lobe pathology is to examine their patterns of 
performance on tasks that have been used with frontal patients. One such study is that of Gershberg and Shimamura (1995) who asked patients with frontal lobe damage and matched typical participants to study lists of 24 categorised words under conditions where category labels were provided at encoding only, retrieval only, encoding and retrieval or neither encoding nor retrieval. In each condition, a first list was studied over a series of study-test trials until either a criterion of $75 \%$ correct recall was reached or until five trials had been completed. After a 5-minute delay, participants were required to study a second list containing different items from the same set of categories as the first list. Results showed that frontal patients took longer to reach criterion in learning the first list, showed marginally improved recall when support was provided at encoding or retrieval but not both, and showed increased relative interference by the first list on recall of the second list. Frontal patients also showed diminished clustering of their recall according to the categories that made up the study lists. They also showed diminished subjective organisation (Tulving 1962) of their recall.

On the basis of the parallels between the patterning of memory in ASD, and that observed in typically ageing individuals and in frontal lobe patients, the aim of the present investigation was to test the hypothesis that individuals with ASD would show similar 'frontal' memory difficulties to those reported by Gershberg and Shimamura (1995) for patients with damage to the frontal lobes. We would predict that in the current experiment individuals with ASD should (1) show slower learning than typical comparison participants, (2) exhibit improved performance when support is provided at either encoding or at retrieval (this would also be predicted on the basis of the TSH), (3) show greater interference from the first list in their learning of a second list, and (4) cluster categorically related items to a lesser extent than comparison participants in recall. Any deviation from this predicted pattern of results, would constrain the use of frontal lobe patients as a heuristic model for investigating memory functioning in ASD and prompt consideration of alternative neuropsychological systems.

\section{Method}

\section{Participants}

Sixteen adults with ASD (3 female, 13 male) and 16 typical individuals ( 3 female, 13 male) took part. Participants from the two groups were individually matched to within 7 points of verbal IQ as measured by the third edition of the Wechsler Adult Intelligence Scale (WAIS-III ${ }^{\mathrm{UK}}$; The Psychological Corporation 2000). Chronological ages,
Table 1 Summary of age and IQ characteristics of the ASD and typical group

\begin{tabular}{|c|c|c|c|c|}
\hline \multirow[t]{2}{*}{ Measure } & \multicolumn{2}{|c|}{$\operatorname{ASD}(n=16)$} & \multicolumn{2}{|c|}{ Typical $(n=16)$} \\
\hline & $M$ & SD & $M$ & SD \\
\hline Age (years) & 35.7 & 13.6 & 34.2 & 12.3 \\
\hline $\mathrm{VIQ}^{\mathrm{a}}$ & 105.5 & 14.9 & 105.9 & 15.2 \\
\hline $\mathrm{PIQ}^{\mathrm{b}}$ & 104.6 & 18.2 & 107.0 & 11.5 \\
\hline $\mathrm{FIQ}^{\mathrm{c}}$ & 103.7 & 16.4 & 106.87 & 14.1 \\
\hline
\end{tabular}

${ }^{\text {a }}$ Verbal IQ (WAIS-R ${ }^{\mathrm{UK}}$ or WAIS-III ${ }^{\mathrm{UK}}$ )

${ }^{\mathrm{b}}$ Performance IQ (WAIS-R ${ }^{\mathrm{UK}}$ or WAIS-III ${ }^{\mathrm{UK}}$ )

${ }^{c}$ Full-Scale IQ (WAIS-R ${ }^{\mathrm{UK}}$ or WAIS-III ${ }^{\mathrm{UK}}$ )

performance, verbal and full-scale IQs for the two groups are set out in Table 1, analysis of which revealed no significant differences between the groups. For the participants with ASD a review of available medical records and/ or assessment with the Autism Diagnostic Observation Schedule (ADOS; LeCouteur et al. 1989) confirmed that all met DSM-IV-TR (APA 2000) criteria for an Autism Spectrum Disorder. ADOS scores were only used as an exclusion criterion when medical records did not provide sufficient detail to confirm that relevant DSM criteria were met. Records of six individuals did not include such details but they all met relevant cut-offs for an ASD on the ADOS assessment. Two individuals whose ADOS scores fell below the cut-off (by 1 point), and three individuals who had not been assessed with the ADOS were included in the present study because their medical records unambiguously suggested that such a diagnosis was appropriate. Participants in the typical comparison group were recruited through local newspaper advertisements and included in the current study only if they were free of psychotropic medication and did not report any family history of neuropathology or psychiatric illness. Informed consent was obtained from all participants and payment for participation was made at standard University rates.

\section{Materials and Design}

Following Gershberg and Shimamura's (1995) third experiment, eight lists of categorized words were constructed. Each of these eight lists consisted of four words from each of six semantic categories for a total of 24 words per list. The category exemplars were chosen from the Battig and Montague (1969) norms and had a mean category rank of 9.8 (range: 1st-29th most popular response) and an average word frequency of 37 per million (range 0-591; Kucera and Francis 1967). The eight lists were constructed in such a way that pairs of lists contained different exemplars from the same semantic category whilst no semantic category was included in more than one list pair. Thus a total of 24 
categories were included ( 6 for each of the four list pairs), the relevant labels for which would serve as the encoding and/or retrieval cues (see section "Procedure").

During the study phase of the experiment, words were presented in bold, Arial, 56-point font in the centre of a Sony Laptop monitor. The order of presentation was pseudorandom with the constraint that no successive pair of words included exemplars from the same semantic category. The rate of presentation was set at 5 seconds per word with no inter-stimulus interval. The category labels that served as encoding and/or retrieval cues were printed in a single column on an A4 sheet in Arial, 56-point font.

\section{Procedure}

Participants were tested individually in a sound attenuated laboratory. All participants were tested on each of four experimental conditions, which will be referred to as the No-cues condition, Encoding-cues condition, Retrievalcues condition and Full-cues condition. In each of these conditions, participants were first required to learn one of the categorised lists of a pair up to a $75 \%$ criterion (i.e. 18 out of 24 words) over a minimum of 2 and a maximum of 5 study-test trials. After a 5-min break, during which participants completed a non-verbal distracter task, participants were required to learn the second of the categorised lists of the relevant pair over 3 study-test trials. The non-verbal distracter task either comprised a digit comparison exercise, the Colour Trails Test or an embedded figures task. The choice of task was random and dictated by the requirements of our participant database. In each of the study-test trials participants were presented with a random sequence of words as described above and immediately after the last word they were asked to free recall orally as many words as possible. During the No-cues condition, no category cues were made available at any stage of the experimental procedure. During the Encoding-cues condition, participants were given a list of relevant category labels during the study phase and were asked to state the category membership of each word as it appeared on the screen. The sheet of category labels was removed as soon as the last word disappeared from the screen and was thus not available whilst participants attempted to free recall. During the Retrievalcues condition, relevant category labels were made available to participants as they attempted to free recall but not whilst the words appeared on the screen and during the Fullcues condition, a list of relevant category labels was available throughout the study-test trials.

For logistical reasons participants completed the four experimental conditions over two separate testing sessions rather than four as in the original Gershberg and Shimamura (1995) study. Our counterbalancing strategy was identical to the original design however. Thus on the first testing session all participants first completed the No-cues condition followed by either the Encoding-cues or the Retrievalcues condition. On the second testing session participants proceeded with either the Encoding-cues or Retrieval cues condition (depending on which was still to be completed), and ended the experiment with the Full-cues condition. Within each session, the two experimental conditions were separated by approximately 1 hour, which was either filled with a lunch break or an unrelated experiment. The categorised study lists were fully counterbalanced across participants such that each list appeared equally often as either the first or second list and in each condition.

\section{Results}

In order to rule out the possibility that any between-group differences in recall resulted from increased repetitions, we first tested whether the groups differed in the numbers of words repeated during free recall. A 2 (group) $\times 2$ (1st List vs. 2 nd List) $\times 4$ (Condition) mixed ANOVA of the total number of repetitions across trials ${ }^{1}$ revealed a main effect for Condition $[F(3,28)=4.76, p<.05]$, with participants repeating an average of 2 words during the No-cues condition and less than 1.3 words in all other conditions. None of the effects or interactions involving the Group factor was significant (all $F s<1.5$ ).

To test our first prediction, we carried out an analysis of the number of study-test trials needed to reach the $75 \%$ recall criterion for List 1 . These data are set out in Table 2 and Fisher's exact probabilities (1-sided) for these group differences are .086 for the No-cues condition, .113 for the Retrieval-cues condition, .051 for the Encoding cues condition and .051 for the Full-cues condition. To determine whether groups differed in the average number of trials they required to reach criterion, we assumed that those who did not reach criterion by Trial 5, would have done so by Trial 6 (this is the most conservative assumption possible and so is unlikely to inflate possible group differences). A 2 (group) $\times 4$ (condition) ANOVA of the number of trials to criterion, revealed a marginal effect of condition $[F(3,28)=2.80$, $p=.058]$ but no significant Group effect $[F(1,30)=$ $2.56, n s]$ or interaction $[F(3,28)=.71, n s]$. The ASD group required an average of $3.2(\mathrm{SD}=1.7)$ trials whilst the typical comparison group required an average of 2.5 $(\mathrm{SD}=.8)$ trials.

To test our second prediction that there would be a facilitatory effect of category cue provision on free recall

\footnotetext{
${ }^{1}$ Since there was some variability in the number of study-test trials participants required to reach criterion on the first list studied, Trial was not included as a factor.
} 
Table 2 Numbers of participants failing to reach criterion and average numbers of trials to criterion for both groups in each condition

\begin{tabular}{|c|c|c|c|c|}
\hline \multirow[t]{2}{*}{ Condition } & \multicolumn{2}{|l|}{$\operatorname{ASD}(n=16)$} & \multicolumn{2}{|c|}{ Comparison $(n=16)$} \\
\hline & $\begin{array}{l}n \text { Failing to reach } \\
\text { criterion }\end{array}$ & $\begin{array}{l}\text { Average trials } \\
\text { to criterion }{ }^{\mathrm{a}}\end{array}$ & $\begin{array}{l}n \text { Failing to reach } \\
\text { criterion }\end{array}$ & $\begin{array}{l}\text { Average trials } \\
\text { to criterion }{ }^{\mathrm{a}}\end{array}$ \\
\hline No-cues & 5 & $3.5(2.1)$ & 1 & $2.7(1.2)$ \\
\hline Encoding-cues & 3 & $3.4(1.8)$ & 0 & $2.5(1.0)$ \\
\hline Retrieval-cues & 4 & $2.9(1.9)$ & 0 & $2.6(.7)$ \\
\hline Full-cues & 4 & $3.1(1.9)$ & 0 & $2.2(.8)$ \\
\hline
\end{tabular}

${ }^{a}$ For the computation of the average number of trials to criterion, participants who failed to reach the criterion by trial 5 were assumed to have done so by trial 6

over the first two trials of the first list in each experimental condition, we conducted a 2 (Group) $\times 2$ (Trial) $\times 4$ (Condition) mixed ANOVA on the proportion of correctly recalled words. Similar to Gershberg and Shimamura (1995), this analysis revealed a marginally significant effect of Condition $[F(3,28)=2.39, p=.09]$ with participants performing significantly better on the Full-cues $(M=.64, \mathrm{SD}=.18)$ than the No-cues $(M=.58, \mathrm{SD}=$ $.17)$ condition $(t=2.59, d f=31, p<.05)$, whilst performance on the Encoding-cues $(M=.60, \mathrm{SD}=.18)$ and Retrieval-cues $(M=.60, \mathrm{SD}=.19)$ conditions fell in between. The main effect of Trial was also significant $[F(1,30)=298.90, p<.001]$, which simply confirms that participants' recall performance improved over the two trials. There were no main effects or interactions involving the Group factor suggesting that, unlike the frontal lobe patients studied by Gershberg and Shimamura (1995), individuals with ASD do not exhibit slower learning, nor do they differentially benefit from encoding or retrieval cues. We did observe an interaction between Trial and Condition $(F(3,28)=4.85, p<.01)$. Figure 1 depicts this interaction and shows a difference in improvement over trials between the Encoding-cues $(M=.16, \mathrm{SD}=.13)$ and Retrieval-cues $(M=.25, \mathrm{SD}=.11)$ conditions. More specifically, the availability of category cues during encoding, although helpful on the first trial, does not appear particularly useful in terms of facilitating learning over trials (i.e. on the second trial performance on this condition is nearly identical to the No-cues condition). Provision of category cues during retrieval, on the other hand, seems to facilitate learning whilst not helping on the first trial.

Whilst the analysis presented above revealed no group differences on recall performance on List 1, an analysis of performance on the second list revealed a marked attenuation of learning over trials in the ASD group. More specifically, a 2 (Group) $\times 3$ (Trial) $\times 4$ (Condition) mixed ANOVA of the recall data of List 2 revealed a main effect of Trial $(F$ $(2,29)=246.60, p<.001$, Greenhouse-Geisser correction) and a significant Trial $\times$ Group interaction $[F(2,29)=$ $10.93, p<.001$, Greenhouse-Geisser correction], which, as

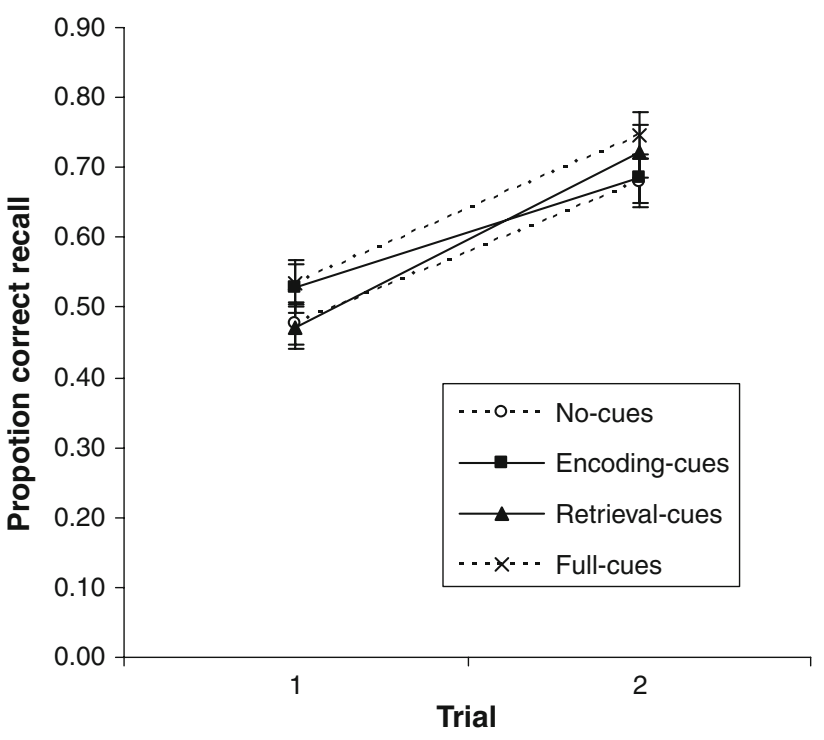

Fig. 1 Proportion of correctly recalled words on the first two trials of List 1 for each experimental condition. Note: For clarity the No-cues and Full-cues conditions are distinguished from the Encoding-cues and Retrieval-cues conditions in order to highlight the interaction between the latter two

Fig. 2 suggests, reflected the ASD group's improving significantly less $(M=.25, \mathrm{SD}=.12)$ over the three trials than the typical comparison group $(M=.38, \mathrm{SD}=.07) .^{2}$ No other main effects or interactions were significant thus replicating the finding by Gershberg and Shimamura (1995) that the provision of category cues has little effect (at least for typical participants) for studying a second categorised list.

In order to determine to what extent the above interaction between Trial and Group was a reflection of group differences in memory interference effects, we first conducted an analysis of the number of intrusions of List 1 words during

\footnotetext{
2 These means and standard deviations refer to the average difference across conditions in the proportion of words recalled between Trial 1 and Trial 3. The trial factor has three levels in this analysis because all participants completed 3 study-test trials for the second list studied. An analysis of the data across only the first two trials yields the same pattern results.
} 


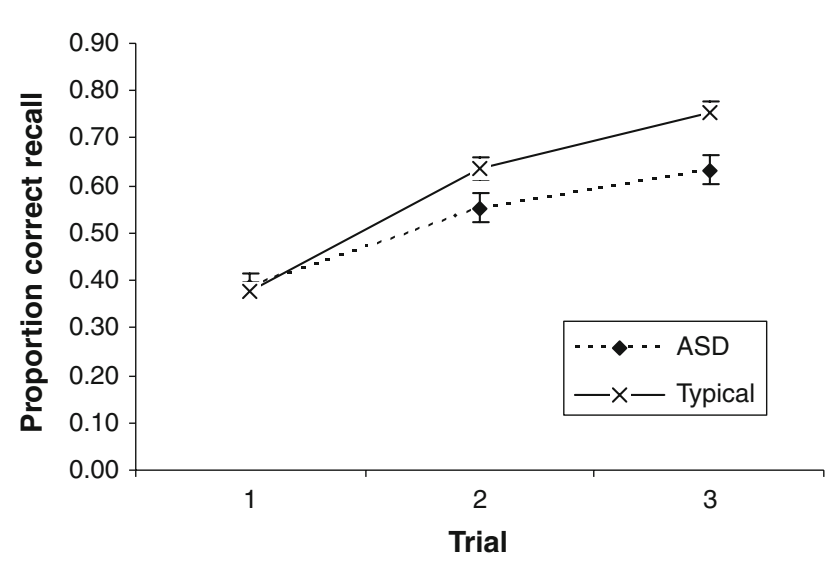

Fig. 2 Average proportions of correctly recalled words on the three trials of List 2 for each participant group

the recall of List 2. A 4 (Condition) $\times 2$ (Group) mixed ANOVA of these intrusions revealed a marginal effect of Condition $[F(3,28)=2.49, p=.08]$ but no effect of Group or interaction between the factors. The effect of Condition resulted from somewhat more frequent intrusions during the Full-cues $(M=4.50, \mathrm{SD}=3.64)$ than the Nocues $(M=3.22, \mathrm{SD}=2.89)$ condition, whilst intrusions for the Encoding-cues $(M=3.78, \mathrm{SD}=2.81)$ and Retrieval-cues conditions $(M=3.50, \mathrm{SD}=3.14)$ conditions fell in between these values. A more sensitive measure of memory interference is the degree to which recall of List 2 is attenuated in comparison to recall of List 1 on Trial 1 of each list. In order to assess this first trial interference, we computed absolute and relative difference scores between performance on Trial 1 of the first and second list. Since these analyses revealed similar patterns of results and because groups performed similarly on the first list studied, we only present results relating to the relative difference scores as these more stringently control for variability in recall performance. A 2 (Group) $\times 4$ (Condition) mixed ANOVA of these data revealed no main effects or interactions $(F \mathrm{~s}<2.3, p \mathrm{~s}>.1)$. Thus, although individuals with ASD showed attenuated learning of List 2 (see analysis above), there was no indication of increased first trial interference effects.

Our final set of analyses tests our fourth prediction and concerns the extent to which participants clustered members of the same category during their free recall attempts. For this purpose we computed the Adjusted Ratio of Clustering (ARC; Roenker et al. 1971), which ranges from -1.0 to 1.0 (chance level $=0$ ) and indices the extent to which successively recalled words stem from the same semantic category. A 2 (group) $\times 2$ (Trial) $\times 4$ (Condition) ANOVA of these data for the first list studied revealed a main effect of Condition $(F(3,28)=4.92, p<.01)$, a marginally significant effect of Trial $(F(1,30)=3.21, p=.08)$ a Condition $\times$ Trial interaction $(F(3,28)=3.42, p<.05)$ and a significant effect of $\operatorname{Group}(F(1,30)=5.00, p<.05)$. The main effect of Condition reflected participants' significantly lower clustering during the No-cues $(M=.57 ; \mathrm{SD}=.39)$ than the Encoding-cues $(M=.74 ; \mathrm{SD}=.31 ; t=2.50$, $d f=31, p<.05)$ and Full-cues conditions $(M=.80$, $\mathrm{SD}=.29 ; t=3.90, d f=31, p<.001)$, whilst the interaction between Condition and Trial was a reflection of clustering scores increasing over trials only for the No-cues $(t=2.17, d f=31, p<.05)$ condition. The absence of a Group by Condition interaction shows that clustering by both groups benefitted to a similar extent from the provision of cues, and thus confirms the TSH. Of most interest, however, was the observation of a significant main effect of Group, which reflected the fact that the ASD participants clustered significantly less than typical participants overall (ASD group $M=.60, \mathrm{SD}=.34$; Typical group $M=.80$, $\mathrm{SD}=.12$ ). Together with the finding that ASD participants recalled a similar number of words over the two trials of List 1 (see earlier analysis), the reduced clustering scores in this group would indicate that recall performance is mediated by semantic clustering to a lesser extent than in typical participants. However, analysis of correlations between recall performance and clustering provides only limited support for this impression. There is a significant association between recall and clustering only for the typical group $(r=.69, p<.01)$. The corresponding correlation for the ASD group was not significant $(r=.34, n s)$. However, inspection of scatter plots revealed that one individual with ASD had recall performance that was amongst the best of the entire group whilst his clustering scores were amongst the lowest (questioning of this individual revealed that he employed a visual strategy to remember the items). Exclusion of this participant yielded a significant correlation between average recall performance and clustering scores $(r=.79, p<.001)$ similar to the typical group (averages are across conditions and across the first two trials of List 1).

A similar analysis of clustering data for List 2 yielded no significant effect of Condition, which parallels the findings from the recall data. The main effect of Trial, however, $[F(1,30)=5.16, p<.05]$ was significant and although the main effect of group was marginally significant $[F(1,30)=3.47, p=.07$; ASD group $M=.57, \mathrm{SD}=.38$; Typical group $M=.76, \mathrm{SD}=.19]$, we did observe a threeway Group $\mathrm{x}$ Trial $\mathrm{x}$ Condition interaction $[F(3,28)=$ $4.16, p<.05]$. Further inspection of the data revealed no clear pattern for this interaction. More specifically, whilst individuals with ASD clustered less than the typical group on Trial 1 of the Full-cues condition (ASD group $M=.40$, $\mathrm{SD}=.56 ;$ Typical group $M=.88, \mathrm{SD}=.21 ; t=3.21$; $d f=30 ; p<.01$ ), on Trial 2 the difference lay mainly in the Retrieval-cues condition (ASD group $M=.60, \mathrm{SD}=.42$; Typical group $M=.90, \mathrm{SD}=.15 ; t=2.70 ; d f=30$; $p<.05)$. Since the clustering scores generally varied 
widely within participants across trials and conditions, we do not attach too much significance to this interaction effect. The important aspect of the data to note is that as a group, ASD participants tended to cluster less than the comparison group. Once again, an analysis of correlations between recall performance and clustering revealed significant associations for the typical $(r=.77, p<.001)$ but not the ASD group $(r=.23, n s)$. As for List 1 , inspection of the data revealed an outlier in the ASD group, exclusion of whom resulted in a correlation of $r=.70, p<.01$ for this group.

In addition to assessing participants' clustering scores, we also computed the Pairwise Frequency (PF) measure of subjective organisation (Sternberg and Tulving 1977), which indexes the degree to which participants recall words in similar order on two successive recall attempts. For the 24-item lists employed in the current study the maximum PF score would be 21.08 whereas a score of 0 would indicate chance organisation. A 2 (Group) $\times 4$ (Condition) mixed ANOVA of PF yielded no significant main effects or interactions for either List 1 or List 2. The average PF score across lists for the ASD group was $1.54(\mathrm{SD}=2.08)$ and for the Comparison group $1.34(\mathrm{SD}=.74)$. Both of these values are significantly above the chance level score of 0 (ASD group: $t=2.96, d f=15, p<.05$; Comparison group: $t=7.28, d f=15, p<.05)$. An analysis of correlations between the PF measure and average recall performance over the two trials of each list, again showed significant associations in both groups (List 1: ASD group $r=.81, p<.001$; Typical group $r=.65, p<.01$; List 2 : ASD group $r=.78, p<.001$; Typical group $r=.71$, $p<.01)$. These correlations are unconfounded by any statistical outliers.

\section{Discussion}

The primary aim of this study was to test a frontal hypothesis of memory difficulties in ASD by exploring the effect of provision of cues at encoding and/or retrieval on multiple-trial free recall of lists of words by such individuals. We predicted that the performance of ASD participants would parallel that observed in a previous study (Gershberg and Shimamura 1995) of frontal lobe patients. More specifically, we tested whether individuals with ASD would (a) exhibit slower list learning, (b) show differential performance on conditions in which encoding or retrieval support was provided, (c) demonstrate increased interference and (d) have attenuated levels of category clustering.

Our first prediction was at best marginally supported by the findings reported here. The number of trials needed to learn the first list to criterion did not differ significantly between groups. At best, there was a tendency for ASD participants to fail more frequently to reach the $75 \%$ recall criterion. However, learning of the second list was significantly diminished in the ASD group. Although it is unclear why individuals with ASD exhibited more pronounced difficulties in learning the second as compared to the first list, this finding, taken together with other studies of free recall learning in ASD (e.g., Bowler et al. 2008) suggests that diminishing recall on multi-trial procedures is a robust characteristic of the disorder.

The second prediction-that individuals with ASD would be differentially affected by the provision of support at different stages of the experiment-was at best marginally borne out by the findings. The only significant enhancement of recall emerged when cues were provided at both encoding and retrieval and in this respect, the ASD and comparison participants did not differ. The effectiveness of cue provision at encoding only or retrieval only was limited to the first and the second trial respectively, an effect that was common to both participant groups. In relation to the TSH, which asserts that people with ASD should show better memory under conditions where support for retrieval is provided, the present findings show only limited confirmation since they do not indicate that people with ASD are more likely than typical participants to rely on task support. This conclusion must be tempered by the overall marginal effect of cue provision, which suggests that the design of the present investigation may not have been sufficiently powerful to reveal any group differences in the effects of provision of task support on recall.

In relation to our third prediction - that individuals with ASD would exhibit more marked memory interferenceour observations did not support a parallel between frontal lobe pathology and ASD. Both groups of participants showed interference from the first list on learning of the second list, but the extent of this was similar for both groups. In addition, there was no between-group difference in the number of List 1 intrusions into the recall of List 2, nor of repetitions in recall of either list. One may object to this observation on the basis that individuals with ASD exhibited diminished learning of List 2 as compared to List 1 , which may indicate interference effects. Although this may be the case, our main concern here is to establish to what extent patterns of memory difficulty in ASD parallel those observed in frontal lobe patients. Thus, even if the diminished learning of List 2 in ASD is partially attributable to interference effects, these interference effects would be qualitatively different from those observed in frontal lobe patients, who exhibit disproportionate interference effects already on Trial 1 of List 2 . Furthermore, the existing literature on repetitions and intrusions in the free recall of individuals with ASD is inconclusive. Absence of ASDcomparison group differences in repetitions is reported by Bennetto et al. (1996), Minshew et al. (1992) and Minshew 
and Goldstein (1993), but is reported by Bowler et al. (2000). Elevated intrusions from earlier-learned lists are reported by Bennetto et al. (1996), Bowler et al. (2000) but not by Minshew et al. (1992) or Minshew and Goldstein (1993). All these studies apart from Bowler et al., employed the California Verbal Learning Test (Delis et al. 1986), and all apart from Bennetto et al. (1996) tested adults whose mean verbal IQs were in excess of 90 . These procedural and participant differences may partly explain the variation in the findings. As intrusions and repetitions are both associated with frontal lobe damage (Cermak et al. 1974; Moscovitch 1992; Stuss et al. 1982), their absence here coupled with the variable findings of earlier studies places limits on the extent to which we can draw parallels between memory in ASD and in frontal lobe damage.

The absence of any between-group differences in the single-trial recall of categorised lists or in the role of support on recall contrasts with the group differences in clustering in recall. The ASD group showed significantly less organisation of their recall output into the categories from which the studied items were drawn than did comparison participants. This finding, together with the significant Condition by Trial interaction and the absence of any Group by Condition interaction in the analysis of the clustering data justifies the conclusion that both groups' relative rate of clustering benefited equally from the provision of cues. The absence of a group difference in recall coupled with its presence in clustering suggests that the way in which cues operated to enhance recall may have differed in the two groups. It may be the case that the ASD participants, because of a diminished capacity for relational processing, used category label cues to generate smaller numbers of recall items than did the comparison participants. Diminished relational processing would still enable some benefit to be gained from the provision of cues but in a manner that was less likely to promote clustered recall. Evidence from other studies has demonstrated diminished relational processing in ASD. Gaigg et al. (2008) found that the recall of word lists by high-functioning individuals with ASD showed greater enhancement when participants were asked to perform a pleasantness rating task at encoding than when asked to sort words into categories. The rating task promoted item-specific processing whereas the sorting task promoted relational processing (Hunt and Seta 1984). Gaigg et al. (2008) concluded from these findings that people with ASD experience greater difficulties with relational rather than item-specific processing. Such a difficulty is likely to result an item-by-item cue-use and would yield the kind of enhanced performance in response to cue provision accompanied by diminished item clustering found in the ASD participants of this study. In this context, it should be noted that the performance of the ASD group was more variable than that of the comparison participants. In particular, there was one participant who had the lowest clustering yet the highest recall score. Such variability needs to be included in the development of theoretical frameworks; inclusion of this participant markedly affected the reported association between clustering and recall and serves to show how a possible deficiency (diminished clustering) need not necessarily result in a behavioural impairment (diminished recall) at least for some individuals with ASD.

A final aspect of the present study is the extent to which its findings help to inform us on the possible role of frontal lobe dysfunction on the patterning of memory in individuals with ASD. The relatively spared learning of List 1 suggests that the posterior left and posterior medial dorsolateral frontal regions are not involved in the memory difficulties of people with ASD, since damage to these areas have been shown to produce deficits in free recall (Alexander et al. 2003; Stuss et al. 1994). In addition, the ASD participants did not benefit to a greater extent than did comparison participants from the provision of category cues at encoding and/or retrieval, something which has been reported for patients with frontal damage (Dimitrov et al. 1999; Gershberg and Shimamura 1995; Incisa della Rocchetta 1993) and more particularly with right lateral damage (Turner et al. 2007).

In common with Gershberg and Shimamura's (1995) frontal patients the ASD participants showed diminished categorical clustering of items in recall. Diminished categorical clustering is not a consistent feature of recall in patients with frontal lobe damage. Studies using blocked categorical lists have failed to find diminished clustering (Gershberg and Shimamura 1995; Stuss et al. 1994, Turner et al. 2007) whereas studies using unblocked lists report diminished clustering Baldo et al. 2002, but see Alexander et al. 2003). It is likely therefore that the diminished clustering found in the ASD group in the present study may be the result of diminished relational encoding (see Bowler and Gaigg 2008 for review) resulting from hippocampallymediated processes rather than frontally-mediated difficulties. Finally, unlike frontal patients in other studies (Eslinger and Grattan 1994; Gershberg and Shimamura 1995), the ASD participants here did not show diminished subjective organisation in recall. This contrasts with the finding of Bowler et al. (2008) who report marginally diminished subjective organisation on a free recall learning task. However, that study required recall of 16 items presented in a different order over 16 trials. Moreover, lower subjective organisation in ASD participants was found only for written, not for oral recall. In this respect, the findings reported here point to a lesser involvement of frontal dysfunction in the organisation of recall in individuals with ASD.

The overall picture that emerges from the findings of the present study is that only limited parallels can be drawn 
between the pattern of memory differences seen in ASD and in patients with frontal lobe damage. Recall by individuals with ASD of categorised lists of words is enhanced by the provision of category cues at encoding and recall, but only to a similar extent to that found in typical comparison participants. The degree to which they cluster items in recall is also susceptible to the provision of category cues, but in contrast to their level of overall recall, their clustering is diminished in comparison to that of typical individuals and is less strongly correlated with overall rates of recall. Individuals with ASD also show significantly diminished recall on later trials of a second list possibly because of their reduced ability to organise items categorically. Diminished clustering may play a role in reduced learning of later lists, but does not impact on levels of subjective organisation. The limited frontal involvement in free recall in individuals with ASD revealed by the present findings suggests that we look elsewhere, for example to the role of hipocampallymediated relational processing difficulties to explain memory difficulties in this group. It may be that hippocampally-mediated difficulties feed information to the frontal lobes in a manner that is inadequate for the strategic processing that is needed for efficient learning and retention of complex material. In this respect, the memory difficulties experienced by individuals with ASD may be the result of frontal dysfunction consequent on impaired medial-temporal processes such as relational binding.

Acknowledgments The work reported in this paper was supported by a project grant from the Wellcome Trust to the first and third authors. An earlier report of the work was presented to the International Meeting for Autism Research, Montreal, May 2006.

Open Access This article is distributed under the terms of the Creative Commons Attribution Noncommercial License which permits any noncommercial use, distribution, and reproduction in any medium, provided the original author(s) and source are credited.

\section{References}

Alexander, M. P., Stuss, D. T., \& Fansabedian, N. (2003). California Verbal Learning Test: Performance by patients with focal frontal and non-frontal lesions. Brain, 126, 1493-1503.

American Psychiatric Association. (2000). Diagnostic and statistical manual of mental disorders, fourth edition-text revision. Washington, DC: APA.

Baldo, J. V., Delis, D., Kramer, J., \& Shimamura, A. P. (2002). Memory performance on the California Verbal Learning Test-II: Findings from patients with focal frontal lesions. Journal of the International Neuropsychological Society, 8, 539-546.

Baldo, J. V., \& Shimamura, A. P. (2002). Frontal lobes and memory. In A. D. Baddeley, M. D. Kopelman, \& B. A. Wilson (Eds.), Handbook of memory disorders (pp. 363-379). Chichester: Wiley.

Battig, W. F., \& Montague, W. E. (1969). Category norms for verbal items in 56 categories: A replication and extension of the
Connecticut category norms. Journal of Experimental Psychology Monographs, 80, 1-46.

Bennetto, L., Pennington, B. F., \& Rogers, S. J. (1996). Intact and impaired memory function in autism. Child Development, 67, $1816-1835$

Boucher, J., \& Warrington, E. K. (1976). Memory deficits in early infantile autism: Some similarities to the amnesic syndrome. British Journal of Psychology, 67, 73-87.

Bowler, D. M., \& Gaigg, S. B. (2008). Memory in ASD: Emerging themes and future prospects. In J. Boucher \& D. M. Bowler (Eds.), Memory in autism: Theory and evidence. Cambridge: Cambridge University Press.

Bowler, D. M., Gaigg, S. B., \& Gardiner, J. M. (2008). Subjective organisation in the free recall of adults with Asperger's syndrome. Journal of Autism and Developmental Disorders, 38, 104-113.

Bowler, D. M., Gardiner, J. M., Grice, S., \& Saavalainen, P. (2000). Memory illusions: False recall and recognition in high functioning adults with autism. Journal of Abnormal Psychology, 109, 663-672.

Bowler, D. M., Matthews, N. J., \& Gardiner, J. M. (1997). Asperger's syndrome and memory: Similarity to autism but not amnesia. Neuropsychologia, 35, 65-70.

Cermak, L. S., Butters, N., \& Moreines, J. (1974). Some analyses of the verbal encoding deficit of alcoholic Korsakoff patients. Neuropsychologia, 9, 307-315.

Craik, F. I. M., Morris, L. W., Morris, R. G., \& Loewen, E. R. (1990). Relations between source amnesia and frontal lobe functioning in older adults. Psychology and Aging, 5, 148-151.

Delis, D. C., Kramer, J. H., Kaplan, E., \& Ober, B. A. (1986). The California Verbal Learning Test-research edition. New York, NY: The Psychological Corporation.

Dimitrov, M., Granetz, J., Peterson, M., et al. (1999). Associative learning impairments in patients with frontal lobe damage. Brain and Cognition, 41, 213-230.

Eslinger, P. J., \& Grattan, L. M. (1994). Altered serial position learning after frontal lobe lesion. Neuropsychologia, 32, 729-739.

Gaigg, S. B., Gardiner, J. M., \& Bowler, D. M. (2008). Free recall in autism spectrum disorder: The role of relational and item-specific encoding. Neuropsychologia, 46, 986-992.

Gershberg, F. B., \& Shimamura, A. P. (1995). Impaired use of organizational strategies in free recall following frontal lobe damage. Neuropsychologia, 32, 729-739.

Hermelin, B., \& O'Connor, N. (1967). Remembering of words by psychotic and subnormal children. British Journal of Psychology, $58,213-218$.

Hill, E. L. (2004). Evaluating the theory of executive dysfunction in autism. Developmental Review, 24, 189-233.

Hunt, R. R., \& Seta, C. E. (1984). Category size effects in recall: The role of relational and item-specific information. Journal of Experimental Psychology: Learning, Memory and Cognition, 10, 454-464.

Incisa della Rocchetta, A. I. (1993). Classification and recall of pictures after unilateral frontal or temporal lobectomy. Cortex, $22,189-211$.

Kucera, H., \& Francis, W. N. (1967). Computational analysis of present-day american english. Providence, RI: Brown University Press.

LeCouteur, A., Rutter, M., Lord, C., Rios, P., Robertson, S., Hgrafer, M., et al. (1989). Autism diagnostic interview: A standardised investigator-based instrument. Journal of Autism and Developmental Disorders, 19, 363-387.

Leekam, S., \& Lopez, B. (2003). Do children with autism fail to process information in context? Journal of Child Psychology and Psychiatry, 44, 285-300.

Lezak, M. D. (1983). Neuropsychological assessment (2nd ed.). New York: Oxford University Press. 
Loveland, K. A., Bachevalier, J., Pearson, D. A., \& Lane, D. M. (2008). Fronto-limbic functioning in children and adolescences with and without autism. Neuropsychologia, 46, 49-62.

Minshew, N. J., \& Goldstein, G. (1993). Is autism an amnesic disorder? Evidence from the California Verbal Learning Test. Neuropsychology, 7, 209-216.

Minshew, N., Goldstein, G., Muenz, L. R., \& Payton, J. (1992). Neuropsychological functioning in nonmentally retarded autistic individuals. Journal of Clinical and Experimental Neuropsychology, 14, 749-761.

Moscovitch, M. (1992). Memory and working-with-memory: A component process model based on modules and central systems. Journal of Cognitive Neuroscience, 4, 257-267.

Roenker, D., Thompson, C. P., \& Brown, S. C. (1971). Comparison of measures for the estimation of clustering in free recall. Psychological Bulletin, 76, 45-48.

Smith, B. J., Gardiner, J. M., \& Bowler, D. M. (2007). Deficits in free recall persist in Asperger's syndrome despite training in the use of list-appropriate learning strategies. Journal of Autism and Developmental Disorders, 37, 445-454.
Sternberg, R. J., \& Tulving, E. (1977). The measurement of subjective organization in free recall. Psychological Bulletin, 84, 539-556.

Stuss, D. T., Alexander, M. P., Palumbo, C. L., Buckle, L., Sayer, L., \& Pogue, J. (1994). Organizational strategies of patients wit unilateral or bilateral frontal lobe injury in word list learning tasks. Neuropsychology, 8, 355-373.

Stuss, D. T., Kaplan, E. F., Benson, D. F., Weir, W. S., Chiulli, S., \& Sarazin, F. F. (1982). Evidence for the involvement of orbitofrontal cortex in memory functions as an interference effect. Journal of Comparative and Physiological Psychology, 96, 913-925.

Tager-Flusberg, H. (1991). Semantic processing in the free recall of autistic children: Further evidence for a cognitive deficit. British Journal of Developmental Psychology, 9, 417-430.

Tulving, E. (1962). Subjective organisation in the free recall of "unrelated" words. Psychological Review, 69, 344-354.

Turner, M. S., Cipolotti, L., Yousry, T., \& Shallice, T. (2007). Qualitatively different memory impairments across frontal lobe subgroups. Neuropsychologia, 45, 1540-1552. 